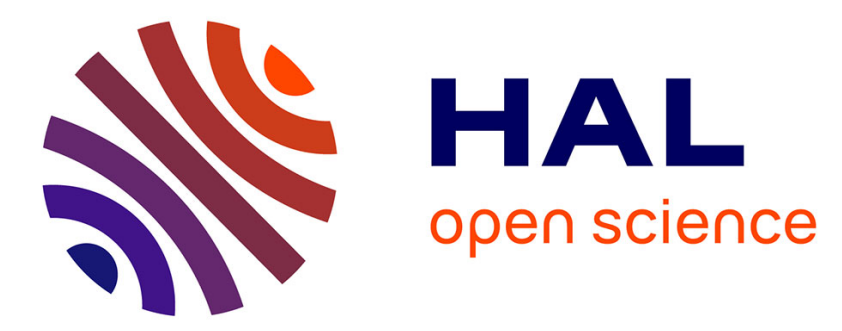

\title{
A NEUTRON SCATTERING STUDY OF THE DENSITY OF VIBRATIONAL STATES OF FRACTAL SILICA AEROGELS
}

\author{
G. Coddens, R. Vacher, T. Woignier, J. Pelous, E. Courtens
}

\section{- To cite this version:}

G. Coddens, R. Vacher, T. Woignier, J. Pelous, E. Courtens. A NEUTRON SCATTERING STUDY OF THE DENSITY OF VIBRATIONAL STATES OF FRACTAL SILICA AEROGELS. Journal de Physique Colloques, 1989, 50 (C4), pp.C4-151-C4-156. 10.1051/jphyscol:1989424 • jpa-00229500

\section{HAL Id: jpa-00229500 https://hal.science/jpa-00229500}

Submitted on 1 Jan 1989

HAL is a multi-disciplinary open access archive for the deposit and dissemination of scientific research documents, whether they are published or not. The documents may come from teaching and research institutions in France or abroad, or from public or private research centers.
L'archive ouverte pluridisciplinaire HAL, est destinée au dépôt et à la diffusion de documents scientifiques de niveau recherche, publiés ou non, émanant des établissements d'enseignement et de recherche français ou étrangers, des laboratoires publics ou privés. 


\title{
A NEUTRON SCATTERING STUDY OF THE DENSITY OF VIBRATYONAL STATES OF FRACTAL SILICA AEROGELS
}

\author{
G. CODDENS $(1)$, R. VACHER*, T. WOIGNIER*, J. PELOUS* and E. COURTENS* \\ Laboratoire Léon Brtllouin, (2) CEN-Saclay, F-91191 Gif-sur-Yvette, \\ France \\ * Laboratoire de science des Matérlaux Vitreux, (3) Université des \\ sciences et Techniques du Languedoc, F-34060 Montpellier, France \\ * IBM Research Division, Zurich Research Laboratory, CH-8803 \\ Rüschlikon, Switzerland
}

\begin{abstract}
Résumé - Après avoir comparé les différentes méthodes couramment utilisées pour mesurer la densité d'états par diffusion inélastique des neutrons, nous présentons des spectres de diffusion purement incohérente obtenus sur des aérogels de silice, à partir de la différence entre les intensités diffusées par des échantillons identiques, protonés et deutérés. Ces résultats concernent les modes de particules et la région de haute fréquence du régime des fractons. Associées à J'information obtenue par diffusion Brillouin, ces mesures donnent l'allure générale de la densité d'états en valeur absolue, et permettent de calculer les propriétés thermiques à basse température, en excellent accord avec les résultats expérimentaux.
\end{abstract}

Abstract - The various methods to measure the density of states by neutron inelastic scattering are discussed. Purely incoherent scattering data on silica aerogels have been obtained by taking the difference spectra between protonated and identical deuterated samples. The results so far cover the particles modes and the upper part of the fracton regime. Complemented with Brillouin scattering information, these measurements provide an overall picture of the absolute density of states, from which low temperature thermal properties can be calculated, in remarkable agreement with experiments.

\section{1 - INTRODUCTION}

The theory of vibrational excitations in fractal structures predicts the existence of "fractons" /1/. Those vibrational modes are localized. Also, in contrast to long-wavelength acoustic phonons, which obey a linear dispersion law, $\omega=\vee \mathrm{q}$, where $\omega$ is the frequency, $q$ is the wavevector, and $v$ is the constant sound velocity, fractons are expected to exhibit dispersion according to $\omega \propto \ell \sim \mathrm{D} / \overline{\bar{d}} / 1,2 /$. Here $D$ is the fractal (Hausdorff) dimension, and $\ell$ is a characteristic length for the fracton. Finally, the density of states (DOS) of fractons $Z(\omega)$ was predicted to be proportional to $\omega \overline{\bar{d}}-1 / 1 /$. The dimensions obey the inequality $d \geq D>\overline{\bar{d}}$, with $d$ the Euclidean dimension. As an example, for 3-d percolation clusters and scalar waves the values are $D \simeq 2.5$ and $\overline{\bar{d}}=4 / 3$. The above predictions are at strong variance with the well-known behavior of acoustic phonons, and the differences should be observable experimentally.

Silica aerogels are ideally suited for the experimental study of fracton dynamics. They are porous solid materials. Large pieces can be prepafed, so that their elastic properties can be measured by static as well as by ultrasonic techniques. The optical transparency of aerogels in a broad range of densities and preparation conditions allows the study of their vibrational dynamics by Brillouin and Raman light scattering.

(1) Supported by Neutron Scattering Project IIKW, Natuurkunde, University of Antwerp, B-2610 Wilrijk, Belgium

(2) Laboratoire mixte CNRS-Commissariat à l'Energie Atomique:

(3) Laboratory associated to the Centre National de la Recherche Scientifique (CNRS), $N^{\circ} 1119$ 
Structural studies 13,4 / have demonstrated that silica aerogels are made of elementary particles, of typical size $a$, with more or less defined surfaces. Those particles assemble to form fractal networks, up to an average cluster size $\xi$. At length scales above $\xi$, the material is an homogeneous assembly of such clusters. Corresponding to these different length scales, three distinct vibrational regimes are expected, namely, with increasing frequency: phonons, fractons, and particle modes.

A crossover from phonons to fractons at $\omega_{\mathrm{col}} / 2 \pi \simeq 1 \mathrm{GHz}$ has been demonstrated by Brillouin scattering experiments $/ 5 /$. This technique also allowed the determination of the dispersion curve for fractons $/ 6,7 /$, and gave indication for the occurrence of localization at the phonon-fracton crossover. Evidence of an extended fracton domain, ranging approximately from $40 \mu \mathrm{eV}$ to $1 \mathrm{meV}$, was obtained from very-low frequency Raman scattering $/ 8 /$. It is obviously of interest to measure directly. the DOS of these excitations.

\section{2 - EXPERIMENTAL APPROACHES}

There are several ways to measure a vibrational DOS by inelastic neutron scattering. One basic idea is that the DOS is reflected in the incoherently scattered neutrons. Taking only the one phonon terms in a multiphonon expansion, the expression for the double differential incoherent cross section of a Bravais lattice is $/ 9 /$,

$$
\left(\frac{d^{2} \sigma}{d \Omega d E^{\prime}}\right)_{\text {inc }}=\frac{\sigma_{\text {inc }}}{4 \pi} \frac{k^{\prime}}{k} \frac{3 N}{2 M} \exp (-2 W) \frac{\left\langle(\vec{q} \cdot \vec{e})^{2}\right\rangle}{\omega} Z(\omega) n(\omega)
$$

Here, $\Omega$ is the collection angle, $E^{\prime}$ the scattered neutron energy, $\sigma_{\text {inc }}$ the incoherent neutron-scattering cross section of the atom, $\mathbf{k}$ and $\mathbf{k}^{\prime}$ the wave vectors of the incident and scattered neutrons, respectively, and $\mathbf{q}=\mathbf{k}^{\prime}-\mathbf{k}$, the momentum exchange. The number of unit cells in the crystal is $N$, and $M$ is the atomic mass, while $e$ and $\omega$ are the polarization and the frequency of a normal mode, respectively. The symbol $<>_{\text {av }}$ indicates average over all normal modes and $n(\omega)=1 / 2(\operatorname{coth} 1 / 2 \beta \hbar \omega \pm 1)$ is the Bose-Einstein population factor, with $\beta=1 / k_{B} T$ and $k_{B}$ the Boltzmann constant. Finally, $Z(\omega)$ is the density of states and $\exp (-2 W)=\exp \left[-1 / 3 q^{2}<u^{2}>\right]$ is the Debye-Waller factor, $u$ being the atomic displacement. The crystal forces are assumed harmonic, and multiphonon processes are neglected. For polyatomic crystals, a similar expression can be written $/ 10 /$, but only a generalized phonon DOS can be determined because the partial contributions of each element are weighted by their individual cross-sections. The calculation can be extended to amorphous solids by considering the whole solid as one giant unit cell /11/.

A fundamental difficulty in applying (1) to the data lies in the fact that those also contain contributions from coherent scattering, and that it is often impossible to separate the coherent and incoherent parts. The various methods to obtain the DOS are just different philosophies on how one can solve this problem.

1. The incoherent approximation consists in ignoring the coherent contribution. The DOS is obtained from an extrapolation to $\mathrm{q} \rightarrow 0$,

$$
\frac{Z(\omega)}{\omega} \propto \lim _{q \rightarrow 0}\left[s \frac{(q, \omega)}{q^{2}}\right] / n(\omega),
$$

where the incoherent cross-section has been approximated by the scattering function $\mathrm{S}$,

$$
\left(\frac{d^{2} \sigma}{d \Omega d E^{\prime}}\right)_{\text {inc }}=S(q, \omega) \frac{\sigma_{\text {inc }}}{4 \pi} \frac{k^{\prime}}{k} N
$$

The incoherent scattering is expected to be more important at large q-values, while the coherent scattering should be more important at small q. This shows that this extrapolation is somewhat self-contradictory, especially for fractons which have a large scattering at small $q$.

2. Other approaches attempt to take into account the contribution of coherent scattering. We limit our discussion to amorphous solids /12/. In this case, Buchenau /13/ has developed a beautiful method based on a comparison of the elastic and inelastic intensities, which does not require the separation of the coherent and incoherent parts. It is important to outline the underlying assumptions. Since the method starts from the work of Carpenter and Pelizzari /11/, all modes in the giant unit cell must be taken into account to obtain the correct relationship between the DOS and $S(q, \omega)$. In general the neutron experiment is not able to do this 
around $q=0$, since sound waves are propagating faster than the incoming neutrons of energy $E$. In fact, for coherent scattering, three equations must be fulfilled simultaneously, namely the energy ( $\left.\hbar \omega=E-E^{\prime}\right)$ and momentum ( $q=\mathbf{k}-\mathbf{k}^{\prime}$ ) conservation laws of the neutron, and the phonon dispersion relation $\omega=v q$ around $q=0$. It is not always possible to satisfy these three equations simultaneously. The energy and momentum conservation laws define a parabola $\omega=\omega(q, E, \theta)$ in the $(q, \omega)$ plane, where $\theta$ is the scattering angle. The straight line corresponding to the phonon dispersion relation does not always intercept this parabola. In a crystalline solid it is nevertheless possible to find a solution; since periodicity allows to meet the dispersion relation at $q_{n}=\mathbf{q}+\mathbf{G}$ where $q_{n}$ is the neutron momentum exchange, while $\mathbf{G} \neq \mathbf{0}$ is a reciprocal lattice vector. This is the so-called Umklapp scattering process. In Buchenau's method it is assumed that a similar process can also take place in a non-periodic solid, a diffuse Umklapp scattering process. Also in such a case, only a small part of the neutron momentum is used to create a vibrational quantum $\left(q_{n} \geqslant q\right)$. In this process, the broad maxima of the coherent structure factor $s(q)$ are analogous to the sharp Bragg scattering peaks. The method works for metallic glasses, but it appears incomplete for vitreous silica $/ 13,14 /$.

3. Another method is the structure-factor approximation. It is assumed that $S(q, \omega)$ can be factorized in $\omega$ and $q$-dependent parts. Then plots of $S(q, \omega)$ vs $q$ at constant $\omega$ should all fall onto each other up to a scale factor. The validity of the assumption is hard to justify theoretically but can be checked experimentally /15/. Using this for sound waves, the assumption of diffuse Umklapp scattering in Buchenau's method can be tested.

4. In the case of porous materials, in particular for aerogels, one can use an expedient described by Richter and Passell /16/. It consists in covering the internal surface with H-atoms. The large incoherent scattering intensity of $\mathrm{H}$-atoms is expected to reflect the motion of the particles in the aerogel. This is certainly the case for excitations whose characteristic length is larger than the particle size. To remove the coherent scattering contribution, one takes the difference between an H-coated and an uncoated sample. In the case of silica aerogels, the particle surface is naturally covered by hydrogen. It is better then to use as reference a sample in which $H$ has been exchanged with $D$. The difference between the spectra obtained on the hydrogenated and the deuterated samples leaves almost perfectly incoherent data. Further, these data being derived from one atomic species, one obtains the true density of states for the proton motion. However, owing to averaging over many sites, there is a dynamic Debye-Waller factor that can be different from the static one, as we explained elsewhere $/ 17 /$.

\section{3 - RESULTS AND DISCUSSION}

The aerogels used for this study are similar to samples whose preparation and structure have been described previously /4/. Three samples reacted under neutral catalysis were used. They are designated by N095, N185, and N360, respectively, the figures indicating the densities in $\mathrm{kg} / \mathrm{m}^{3}$. The aerogels were oxidized at $500^{\circ} \mathrm{C}$ to remove $\mathrm{CH}_{3}$-groups that could otherwise contribute to the inelastic incoherent signal. The spectra were recorded with the Mibemol time-of-flight spectrometer at the Laboratoire Léon Brillouin in Saclay. The experimental procedure was already described elsewhere $117 /$.

A study of the temperature dependence of the incoherent inelastic scattering has been performed on the N185 sample. Using Eq. (2) to extract $Z(\omega)$, a practically T-independent result is obtained /17/. A weak $T$-dependence could be caused by anharmonicity. Thus, our further discussion focuses on measurements at the lower temperature of $60 \mathrm{~K}$. Results at this temperature for samples N095, N185, and N360 are shown in Fig. 1.

The high-frequency end of the fracton regime is expected at $\omega_{\cos }=\omega_{\operatorname{co1}}(\xi / a)^{D / \overline{\bar{d}}} \propto a^{-D / \bar{d}}$, since $\omega_{\mathrm{co1}} \propto \xi^{-D / \bar{d}}$. The Guinier radii of the particles are in the range of $\sim 6$ to $10 \AA$, from which $\omega_{\mathrm{co} 2}$ in the range of $\sim 1$ to $3 \mathrm{meV}$ is anticipated. The lowest frequency modes of the particles are surface modes for which $\omega \propto a$. They occur at $\sim 3 \mathrm{meV}$ but appear rather broad /8/, presumably owing to the polydispersity in particle size and shape. Hence, the measured $Z(\omega)$ covers the high-frequency fractons and the particle modes. From the Raman data /8/, one expects that the particle modes are about the same for these three samples, and this appears to be confirmed in Fig. 1.

The results on the particle DOS can be compared to a formula which has been derived for the DOS of small spheres $148 /$. This comparison requires no adjustable parameters, except for the absolute value in the measurement, which becomes thus calibrated /17/. The DOS in the phonon regime can be calculated from the Brillouin results. The overall picture that emerges for sample N185 is presented in Fig. 2. 
In the particle regime, the effective slope of the DOS is around 1.5, due to the contribution of both surface $(\propto \omega)$ and bulk $\left(\infty \omega^{2}\right)$ modes. The curve is the fit to the theory of Baltes and Hilf /18/. In the fracton regime, we have traced a line of slope $\overline{\bar{d}}-1=0.3$ through the measured points below $\omega_{\text {co2. }}$ It intercepts the phonon DOS near $\omega_{\text {co1 }}$. So far, the dashed line should be considered as a conjecture. In particular we cannot decide yet whether there is a bump of not in the region of $\omega_{\text {co1 }} / 19 /$. However, since the proposed DOS is on an absolute scale, it can be used to calculate the specific heat $C$ without any adjustable parameters. The behavior which is found $/ 17 /$ is in remarkable agreement with low temperature measurements $/ 20 /$. In particular, recent thermal results on neutrally reacted samples $/ 21 /$ give a curve for $\mathrm{C} / \mathrm{T}^{3}$ which has exactly the same shape as our calculated curve $/ 17 /$ and which agrees with it in absolute value within a factor of 2 . This can be taken as strong evidence that fractons rather than two-level states control the low temperature properties of these materials $/ 22 /$.

The authors thank Prof. J. Teixeira for enlightening discussions.

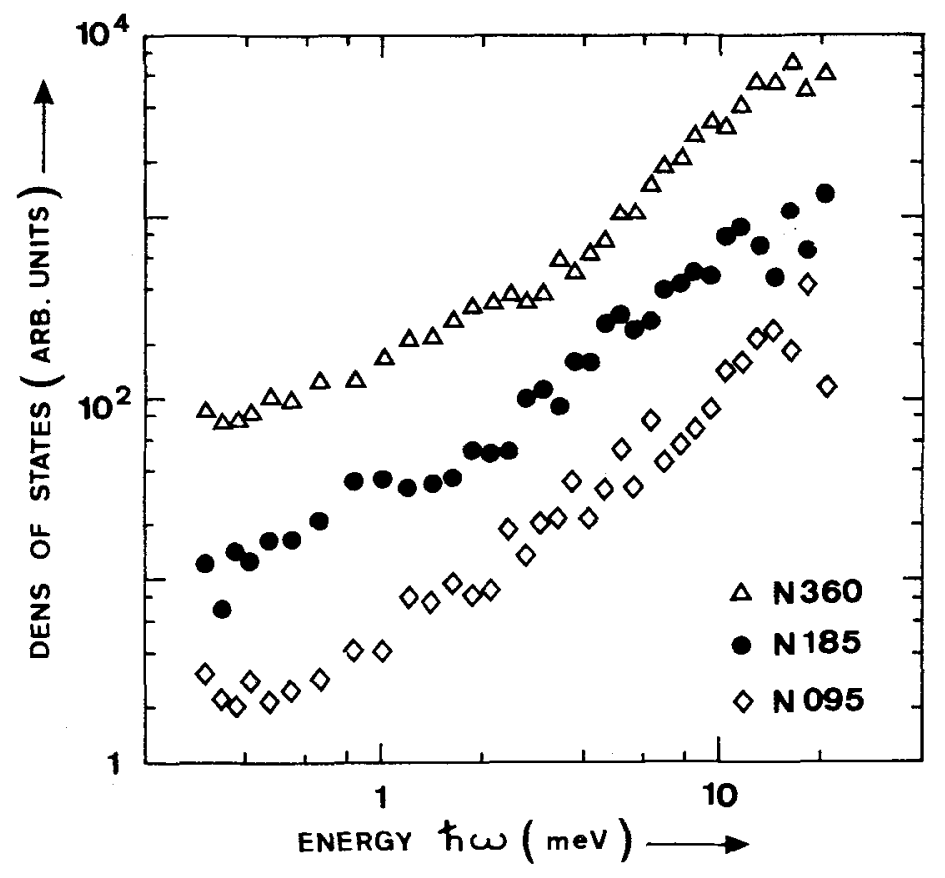

Figure 1 - The measured density of states $Z(\omega)$, in arbitrary units, for three neutrally reacted samples. The numbers in the labels indicate the density in $\mathrm{kg} / \mathrm{m}^{3}$. The measurements were performed at $60 \mathrm{~K}$. 


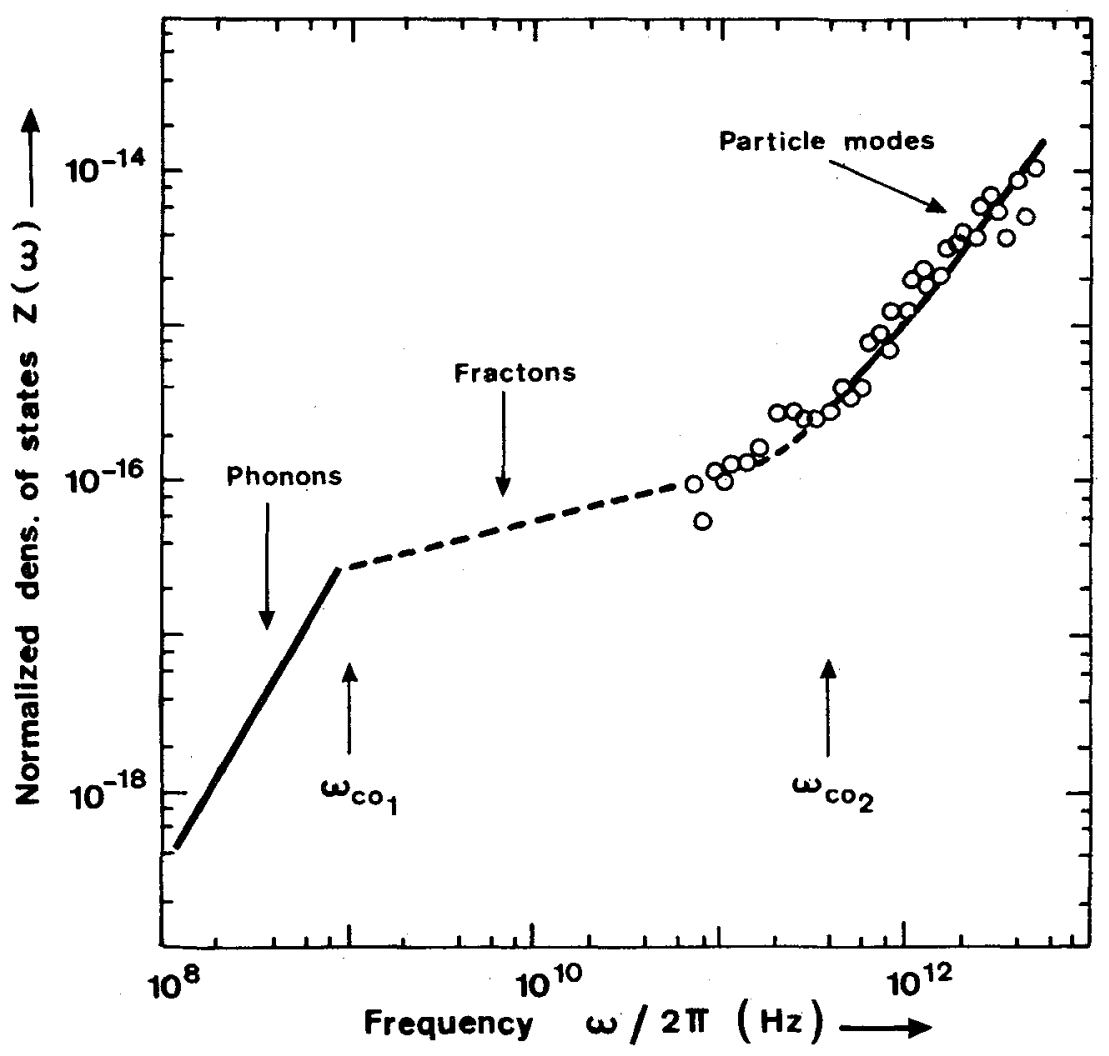

Figure 2. The DOS of the neutrally reacted gel $\mathrm{N} \mathbf{4 8 5}$. The solid line in the phonon regime is the Debye value calculated on the basis of the Brillouin scattering data. The points in the particle region are, up to a vertical displacement, obtained from neutron scattering measurements. The curve is from the theory of Baltes and Hilf $/ 18 /$. The dashed line is the conjectured fracton contribution.

\section{REFERENCES}

1/ Alexander, S. and Orbach, R., J. Phys. (Paris), Lett. 43 (1982) L625.

12/ Rammal, R. and Toulouse, G., J. Phys. (Paris), Lett. 44 (1983) L13.

13/ Schaefer, D. W., Mat. Res. Soc. Bull. 13 (1988) 22.

14/ Vacher, R., Woignier, T., Pelous, J., and Courtens, E., Phys. Rev. B 37 (1988) 6500.

15) Courtens, E., Pelous, J., Phalippou, J., Vacher, R., and Woignier, T., Phys. Rev. Lett. 58 (1987) 128.

16/ Courtens, E., Vacher, R., Pelous, J., and Woignier, T., Europhys. Lett. 6 (1988) 245.

17/ Courtens, E. and Vacher, R.; in Random Fluctuations and Pattern Growth: Experiments and Mode/s, Stanley H. E. and Ostrowski, N., Eds. (Kluwer Academic Publishers, Dordrecht, 1988) p. 21.

18/ Tsujimi, Y., Courtens, E., Pelous, J., and Vacher, R., Phys. Rev. Lett. 60 (1988) 2757.

19/ Squires, G. L., Thermal Neutron Scattering, Cambridge University Press, Cambridge 1978.

/10/ Marshall, W. and Lovesey, S. W., Theory of Thermal Neutron Scattering, Clarendon Press, Oxford 1971.

111/ Carpenter, J. M. and Pelizzari, C. A., Phys. Rev. B 12 (1975) 2391. 
112/ For crystalline solids see e.g. : Gompf, F., Lau, H., Reichardt, W., and Salgado, J., in Proceedings of the Conference on Neutron Ine/astic Scattering (I.A.E.A., Vienna, 1972) p. 137.

/13/ Buchenau, U., Z. Phys. B 58 (1985) 181.

/14/ Buchenau, U., Nücker, N., and Dianoux, A. J., Phys. Rev. Lett. 53 (1984) 2316

/15/ Buchenau, U., Prager, M., Nücker, N., Dianoux, A. J., Ahmad, A., and Phillips, W. A., Phys. Rev. B 34 (1986) 5665 .

/16/ Richter, D. and Passell, L., Phys. Rev. Lett. 44 (1980) 1593; Phys. Rev. B 26 (1982) 4078.

/17/ Vacher, R., Woignier, T., Pelous, J., Coddens, G., and Courtens, E., Europhys. Lett. (to be published).

/18/ Baltes, H. P. and Hilf, E. R., Solid State Commun. 12 (1973) 369.

119/ Aharony, A., Alexander, S., Entin-Wohlman, O., and Orbach, R., Phys. Rev. B 31 (1985) 2565.

/20/ Calemczuk, R., de Goër, A. M., Salce, B., Maynard, R., and Zarembowitch, A., Europhys. Lett. 3 (1987) 1205.

/21/ Maynard, R., Calemczuk, R., de Goër, A. M., Salce, B., Bon, J., and Bonjour, E., in these Proceedings.

/22/ Bon, J., Bonjour, E., Calemczuk, R., and Salce, B., (to be published). 